\title{
Systemic combining inflammatory score (SCIS): a new score for prediction of oncologic outcomes in patients with high-risk non-muscle-invasive urothelial bladder cancer
}

\author{
Matteo Ferro ${ }^{1 \#}$, Marina Di Mauro ${ }^{2 \#}$, Sebastiano Cimino ${ }^{2}$, Giuseppe Morgia ${ }^{2,3}$, Giuseppe Lucarelli ${ }^{4}$, \\ Abdal Rahman Abu Farhan ${ }^{5}$, Mihai Dorin Vartolomei ${ }^{5}$, Angelo Porreca ${ }^{6}$, Francesco Cantiello \\ Rocco Damiano $^{7}$, Gian Maria Busetto ${ }^{8}$, Francesco Del Giudice ${ }^{8}$, Rodolfo Hurle ${ }^{9}$, Sisto Perdonà ${ }^{10}$, \\ Marco Borghesi ${ }^{11}$, Pierluigi Bove ${ }^{12}$, Riccardo Autorino ${ }^{13}$, Nicolae Crisan ${ }^{14}$, Michele Marchioni ${ }^{15}$, \\ Luigi Schips $^{15}$, Francesco Soria ${ }^{16}$, Andrea Mari ${ }^{17}$, Andrea Minervini ${ }^{17}$, Alessandro Veccia ${ }^{12,18}$, \\ Michele Battaglia $^{19}$, Daniela Terracciano ${ }^{20}$, Gennaro Musi ${ }^{1}$, Giovanni Cordima ${ }^{1}$, Matteo Muto ${ }^{21}$, \\ Vincenzo Mirone $^{22}$, Ottavio de Cobelli ${ }^{1 \#}$, Giorgio Ivan Russo ${ }^{2}$
}

${ }^{1}$ Division of Urology, European Institute of Oncology, Milan, Italy; ${ }^{2}$ Urology Section, Department of Surgery, University of Catania, Catania, Italy; ${ }^{3}$ Urology Section, Istituto Oncologico Del Mediterraneo (IOM), Viagrande, Italy; ${ }^{4}$ Department of Urology, Magna Graecia University of Catanzaro, Catanzaro, Italy; ${ }^{5}$ Department of Cell and Molecular Biology, University of Medicine and Pharmacy, Tirgu Mures, Romania; ${ }^{6}$ Department of Urology, Abano Terme Hospital, Padua, Italy; ${ }^{7}$ Department of Urology, Magna Graecia University of Catanzaro, Catanzaro, Italy; ${ }^{8}$ Department of Urology, Sapienza University of Rome, Rome, Italy; ${ }^{9}$ Department of Urology, Istituto Clinico Humanitas Istituto di Ricovero e Cura a Carattere Scientifico-Clinical and Research Hospital, Milan, Italy; ${ }^{10}$ Uro-Gynecological Department, Istituto Nazionale per lo Studio e la Cura dei Tumori, Fondazione "G. Pascale” IRCCS, Naples, Italy; ${ }^{11}$ Department of Urology, University of Bologna, Bologna, Italy; ${ }^{12}$ Division of Urology, Department of Experimental Medicine and Surgery, Urology Unit, Tor Vergata University of Rome, Rome, Italy; ${ }^{13}$ Division of Urology, Virginia Commonwealth University, Richmond, VA, USA; ${ }^{14}$ Department of Urology, University of Medicine and Pharmacy of Cluj-Napoca, ClujNapoca, Romania; ${ }^{15}$ Department of Medical, Oral and Biotechnological Sciences, "SS. Annunziata" Hospital, Urology Unit, Chieti, Italy; ${ }^{16}$ Division of Urology, Department of Surgical Sciences, University of Studies of Torino, Turin, Italy; ${ }^{17}$ Department of Urology, University of Florence, Unit of Oncologic Minimally-Invasive Urology and Andrology, Careggi Hospital, Florence, Italy; ${ }^{18}$ Urology Unit, Department of Medical and Surgical Specialties, Radiological Science, and Public Health, ASST Ospedali Civili, University of Brescia, Brescia, Italy; ${ }^{19}$ Department of Emergency and Organ Transplantation, Urology, Andrology and Kidney Transplantation Unit, University of Bari, Bari, Italy; ${ }^{20}$ Department of Translational Medical Sciences, University of Naples "Federico II", Naples, Italy; ${ }^{21}$ Radiotherapy Unit, "S.G. Moscati" Hospital, Avellino, Italy; ${ }^{22}$ Department of Neurosciences, Sciences of Reproduction and Odontostomatology, Urology Unit, University of Naples "Federico II", Naples, Italy

Contributions: (I) Conception and design: M Ferro; (II) Administrative support: M Ferro; (III) Provision of study materials or patients: All authors; (IV) Collection and assembly of data: All authors; (V) Data analysis and interpretation: GI Russo, Di Mauro, M Ferro; (VI) Manuscript writing: All authors; (VII) Final approval of manuscript: All authors.

\#These authors contributed equally to this work.

Correspondence to: Giorgio Ivan Russo, MD, PhD. Urology Section, Via Santa Sofia 78, 95100, Catania, Italy. Email: giorgioivan1987@gmail.com.

Background: An accurate and early diagnosis of bladder cancer (BC) is essential to offer patients the most appropriate treatment and the highest cure rate. For this reason, patients need to be best stratified by class and risk factors. We aimed to develop a score able to better predict cancer outcomes, using serum variables of inflammation.

Methods: A total of 1,510 high-risk non-muscle invasive bladder cancer (NMIBC) patients were included in this retrospective observational study. Patients with pathologically proven T1 HG/G3 at first TURBT were included. Systemic combined inflammatory score (SCIS) was calculated according to systemic inflammatory markers (SIM), modified Glasgow prognostic score (mGPS), and prognostic nutritional index (PNI) dichotomized (final score from 0 to 3 ).

Results: After 48 months of follow-up (IQR 40.0-73.0), 727 patients recurred (48.1\%), 485 progressed (32.1\%), 81 died for cancer (7.0\%), and 163 died for overall causes (10.8\%). Overall, 231 (15.3\%) patients had concomitant Cis, 669 (44.3\%) patients had multifocal pathology, 967 (64.1\%) patients had tumor size 
$>3 \mathrm{~cm}$. Overall, 357 (23.6\%) patients received immediate-intravesical therapy, 1,356 (89.8\%) received adjuvant intravesical therapy, of which 1,382 (91.5\%) received BCG, $266(17.6 \%)$ patients received mitomycin C, 4 (0.5\%) patients received others intravesical therapy. Higher SCIS was independently predictive of recurrence (hazard ratio HR 1.5, 1.3 and 2.2) and cancer specific mortality for SCIS 0 and 3 (HR: 1.61 and 2.3), and overall mortality for SCIS 0 and 3 (HR: 2.4 and 3.2). Conversely, SCIS was not associated with a higher probability of progression.

Conclusions: The inclusion of the SCIS in clinical practice is simple to apply and can help improve the prediction of cancer outcomes. It can identify patients with high-grade $\mathrm{BC}$ who are more likely to experience disease mortality.

Keywords: Bladder cancer (BC); inflammation; prognosis; outcomes; immune system

Submitted Sep 15, 2020. Accepted for publication Dec 03, 2020.

doi: $10.21037 /$ tau-20-1272

View this article at: http://dx.doi.org/10.21037/tau-20-1272

\section{Introduction}

Bladder cancer (BC) represents one of the most frequent tumor in Europe (1). Fortunately, most of them, about $70 \%$, are in the form of non-muscle invasive bladder cancer (NMIBC), but on the other hand a great part of this may relapse or progress (2). Therefore, an accurate and early diagnosis of NMIBC is essential to offer patients the most appropriate treatment and the highest cure rate. For the same reason, NMIBC patients need to be best stratified by class and risk factors. To this regard, some of them are represented by those from the European Organization for Research and Treatment of Cancer (EORTC) (3) or from the Spanish consortium CUETO (Club Urologico Español de Tratamiento Oncologico) (4). Both risk calculators tend to overestimate the risk of disease recurrence and progression in high-risk patients and present low discrimination for prognostic outcomes in external validation (5).

In a recent research article by Jobczyk et al. (6) it has been shown that EAU, EORTC, and CUETO risk groups appeared to demonstrate moderate performance in the prediction of recurrence and progression, with the one-year and five-year c-indices ranging from 0.55 to 0.66 for recurrence and from 0.72 to 0.82 for progression. Combined with recent advancements in treatment options, those results jointly highlight the urgent need for the development of new stratification tools.

Currently, NMIBC patients are stratified into three risk categories, low-, intermediate- and high-risk, based on the grade, T-stage, tumor size, focality of the tumor, concomitant carcinoma in situ and recurrence rate.
Unfortunately, population-based data have shown that reallife survival is below expectations from clinical trials (7). The need therefore arises to improve the predictive model, improving the stratification in real risk classes and therefore adequately treating the patient. Taking into account these, currently unmet needs and the shortcomings of previous risk stratifications, we aimed to develop a score able to better predict cancer outcomes, using serum variables of inflammation.

We present the following article in accordance with the REMARK reporting checklist (available at http://dx.doi. org/10.21037/tau-20-1272).

\section{Methods}

A total of 1,510 high-risk NMIBC (T1 high grade/G3) subjects from January 1, 2002 and December 31, 2012 were enrolled in this retrospective longitudinal study. All Patients underwent re-TURBT performed within 4-6 weeks and intravesical BCG intravesical therapy. All included patients completed at least 1-year of immunotherapy. Serum data including inflammatory markers were collected 1 month before surgery. Serum inflammatory markers (SIM) was calculated based on previous data (8). The modified Glasgow prognostic score (mGPS) was calculated as described previously (9). Briefly, patients with elevated CRP $(>10 \mathrm{mg} / \mathrm{dL})$ were assigned an mGPS of 1 or 2 depending on the absence or presence of hypoalbuminaemia $(<35 \mathrm{~g} / \mathrm{dL})$, whereas patients showing no elevated level of CRP $(<10 \mathrm{mg} / \mathrm{dL})$ are allocated an mGPS of 0 , even if hypoalbuminaemia is present. The prognostic nutritional 
index (PNI) was calculated as previously reported and dichotomized according the median, attributing a score of 1 to patients with $\mathrm{PNI}<51.55$ (10).

Systemic combined inflammatory score (SCIS) was calculated according to SIM, mGPS and PNI dichotomized cut-offs of the markers. We gave 1 point at SIM $\geq 2$, mGPS $\geq 1$ and PNI ( $<51.55$ ) (range from 0 to 3 ) based on previous literature data (8-10).

Patients with a urinary tract infection or inflammatory systemic diseases that could influence these parameters (chronic intestinal diseases, autoimmune diseases, leukaemia, lymphoma) were excluded from the study. The endpoints of this study were time to recurrent disease, time to progressive disease, overall- and cancer-specific survival (OS and CSS).

The authors are accountable for all aspects of the work in ensuring that questions related to the accuracy or integrity of any part of the work are appropriately investigated and resolved. The study was conducted in accordance with the Declaration of Helsinki (as revised in 2013). The study was approved by institutional ethics board of Policlinico Hospital Federico II of Naples (No.: 310/2018) and informed consent was taken from all the patients.

\section{Statistical analysis}

Continuous variables are presented as the median and interquartile range (IQR). Differences between groups were assessed using a Kruskal-Wallis or Mann-Whitney $\mathrm{U}$ test as appropriate. Categorical variables were tested using a $\mathrm{X}^{2}$ test or Fisher's exact test. Primary outcome of the study was to select variables able to identify recurrence. Secondary outcomes were considered progression, CSS, and OS. For internal validation, the analysis was subjected to 500 bootstrap resamples.

Kaplan-Meier curves and log-rank test were applied. Competing-risks survival regression has been used for recurrence and progression in consideration of the high rate of overall death. ROC (receiver operating curve) was performed to identify accuracy of SCIS, SIM, mGPS and PNI in predicting recurrence.

To perform cross-validation of the models, using resampling techniques for evaluating predictive models, we applied the new command 'cvauroc' of Stata (11). This new command is a Stata rclass program that implements k-fold cross-validation for the AUC for a binary outcome after fitting a logit or probit regression model. Cvauroc averages the AUCs corresponding to each fold and applies the bootstrap procedure to the cross-validated AUC to obtain statistical inference and $95 \%$ bias corrected confidence intervals (CI). Furthermore, cvauroc optionally provides the cross-validated fitted probabilities for the dependent variable or outcome contained in a new variable named fit, the sensitivity and specificity, contained in two new variables named, _sen and _spe, and the plot for the mean cvAUC and k-fold ROC curves (11).

All statistical analyses were carried out using Stata v.14 (StataCorp, College Station, TX, USA). For all comparisons, the significance level was set to $\mathrm{P}<0.05$ for differences between groups.

\section{Results}

Table 1 lists all variables of patients at enrollment. Table S1 shows the relationship between mGPS and variables at enrollment.

After surgery, 375 subjects (24.8\%) had residual highgrade NMIBC and 1,135 (75.2\%) were negative.

We found that subjects with lower mGPS score were more frequent never smoker $(\mathrm{P}<0.01)$, while patients with higher number of cigarettes smoked per day had higher mGPS score $(\mathrm{P}<0.01)$ and patients with longer duration of smoking had also higher mGPS score $(\mathrm{P}<0.01)$. Patients that never used statins had lower mGPS score $(\mathrm{P}<0.02)$. Patients with mGPS of 1 had greater rate of tumor size $\geq 3 \mathrm{~cm}$ than patients with mGPS of $0(70 \% v s$. $62 \%$; $\mathrm{P}=0.02)$. Patients with $\mathrm{mGPS}$ of 1 had greater rate of multifocal disease than patients with mGPS of 0 (51.4\% vs. $41.5 \% ; \mathrm{P}<0.01)$.

Tables S2 and S3 show the relationship between PNI and SIM and variables at enrollment.

Smoking status and duration were associated with higher SCIS. The median cigarettes for day (IQR) was $14(0-25)$ in patients with SCIS 3 vs. 10 (0-20) in patients with SCIS 2. Patients with SCIS of 2 had greater rate of multifocal disease than patients with SCIS of 1 (51.5 vs. 41.5, $\mathrm{P}<0.01$ ) (Table 2).

\section{Oncologic outcomes according to SCIS}

After 48 months of follow-up (IQR 40.0-73.0), 727 patients recurred (48.1\%), 485 progressed $(32.1 \%), 81$ died for cancer $(7.0 \%)$, and 163 died for overall causes (10.8\%).

The median recurrence-free survival was $65[95 \%$ confidence interval (CI): 38.8-91.2], 50 (95\% CI: 42.6-57.35), 36 (95\% CI: 28.7-43.3), and 21 (95\% CI: 
Table 1 Characteristics of the population $(\mathrm{N}=1,510)$

\begin{tabular}{|c|c|}
\hline Characteristic & Variable \\
\hline Age (years), median (IQR) & $71(65.0-78.0)$ \\
\hline \multicolumn{2}{|l|}{ Gender, n (\%) } \\
\hline M & $1,222(80.1)$ \\
\hline $\mathrm{F}$ & $288(18.9)$ \\
\hline \multicolumn{2}{|l|}{ Smoking status, n (\%) } \\
\hline No & $673(44.6)$ \\
\hline Current & $429(28.4)$ \\
\hline Former & $408(27)$ \\
\hline Cigarettes for day, median (IQR) & $10(0-20)$ \\
\hline Smoking (years), median (IQR) & $20(0-30)$ \\
\hline BMI $\left(\mathrm{kg} / \mathrm{m}^{2}\right)$, median (IQR) & $27(24-29.64)$ \\
\hline \multicolumn{2}{|l|}{ Statin use, n (\%) } \\
\hline No & $1,108(73.4)$ \\
\hline Yes & $402(26.6)$ \\
\hline ESR (mm/h), median (IQR) & $12(8-22)$ \\
\hline Albumin (g/dL), median (IQR) & $4.18(3.9-4.5)$ \\
\hline Neutrophil $\left(\times 10^{3} / \mu \mathrm{L}\right)$, median (IQR) & $5.7(4-9.8)$ \\
\hline Lymphocytes $\left(\times 10^{3} / \mu \mathrm{L}\right)$, median (IQR) & $1.9(1.5-2.5)$ \\
\hline Monocytes $\left(\times 10^{3} / \mu \mathrm{L}\right)$, median (IQR) & $0.55(0.4-0.8)$ \\
\hline Eosinophilic $\left(\times 10^{3} / \mu \mathrm{L}\right)$, median (IQR) & $0.16(0.1-0.28)$ \\
\hline Basophilic $\left(\times 10^{3} / \mu \mathrm{L}\right)$, median (IQR) & $0.03(0.01-0.05)$ \\
\hline Platelets $\left(\times 10^{3} / \mu \mathrm{L}\right)$, median (IQR) & $232.5(192.75-282.25)$ \\
\hline SIM, median (IQR) & $1.0(1.0-2.0)$ \\
\hline \multicolumn{2}{|l|}{ SIM, n (\%) } \\
\hline 0 & $150(10.0)$ \\
\hline 1 & $603(40.1)$ \\
\hline 2 & $610(40.6)$ \\
\hline 3 & $140(9.3)$ \\
\hline \multicolumn{2}{|l|}{ mGPS, median (IQR) } \\
\hline \multicolumn{2}{|l|}{ mGPS, n (\%) } \\
\hline 0 & $1,081(71.6)$ \\
\hline 1 & $387(25.6)$ \\
\hline 2 & $42(2.8)$ \\
\hline PNI, median (IQR) & $51.55(48-56.5)$ \\
\hline $\mathrm{PNI}>51.55, \mathrm{n}(\%)$ & $755(49.5)$ \\
\hline
\end{tabular}

ESR, erythrocyte sedimentation rate; mGPS, modified Glasgow prognostic score; PNI, prognostic nutritional index; SIM, systematic inflammatory marker.
17.1-24.9) months in subjects with SCIS scores of 0,1 , 2 , and 3 , respectively $(\mathrm{P}<0.01, \log$-rank test). In multiple comparisons, between-group differences in recurrencefree survival were statistically significant (all $\mathrm{P}<0.01$ ). The median progression-free survival was 86.0 (95\% CI: 59.1112.9), 78 (95\% CI: 71.3-84.7), 92 (95\% CI: 73.8-110.2), and 90 (95\% CI: 62.8-117.2) months in subjects with SCIS of $0,1,2$, and 3 , respectively $(\mathrm{P}<0.01, \log$-rank test).

The median cancer specific survival was 117.8 (95\% CI: 114.5-118.9), 113.4 (95\% CI 109.2-114.7), 115.2 (95\% CI: 111.2-116.5), and 115.5 (95\% CI: 107.6-118.1), months in subjects with SCIS of $0,1,2$, and 3 , respectively $(\mathrm{P}<0.1)$. The median OS was 112.7, (95\% CI: 107.5-114.3), 105.9 (95\% CI: 100.8-107.6), 108.6, 95\% CI: 103.2-110.3), and 104.6 (95\% CI: 92.8-118.5), months in subjects with SCIS of $0,1,2$, and 3, respectively $(\mathrm{P}<0.02)$ (Figures $1-4)$. Figure $\mathrm{S} 1$ shows Fine-Gray competing risk analysis of survival according to SCIS.

In bootstrap-adjusted multivariable Cox regression analyses, increase in SCIS was associated with a significantly higher hazard ratio (HR) for recurrence (HR 1.3, $\mathrm{P}<0.01$; HR 1.55, $\mathrm{P}<0.01$; and HR 2.2, $\mathrm{P}<0.01)$ and cancer specific mortality for SCIS 0 and 3 (HR 1.61, $\mathrm{P}<0.05$; and HR 2.3, $\mathrm{P}<0.01$ ), and overall mortality for SCIS 0 and 3 (HR 2.4, $\mathrm{P}<0.05$; and HR 3.2, $\mathrm{P}<0.05)$. Conversely, SCIS was not predictive of progression (Table 3).

\section{Validation of the predictive model}

After applying the cross-validation (cv) of the models, using resampling techniques (10-fold), we found that for recurrence risk the cvMean AUC (cvStandard Deviation cvSD: 0.06$)$ was 0.552 (95\% CI: $0.49,0.55)$ while for progression risk the cvMean AUC (cvSD AUC: 0.07) was 0.54 (95\% CI: $0.46,0.55$ ). As concerning survival variables, for cancer specific mortality the cvMean AUC (cvSD AUC: $0.1415)$ was 0.5137 (95\% CI: 0.36, 0.52) and for OS was cvMean (cvSD AUC: 0.07) AUC 0.52 (95\% CI: 0.41, 0.52). Figure $\mathrm{S} 2$ shows the ROC for recurrence prediction.

\section{Discussion}

Herein, we showed that a single score based on previous inflammatory scores, denoted as mGPS, PNI and SIM, that we defined as SCIS, is useful in predicting oncological outcomes in patients with high-grade NMIBC.

To this regard, many previous risk tables have been used and are currently applied in these patients to predict 
Table 2 Association between baseline clinicopathologic characteristics and cumulative SCIS score in the total cohort

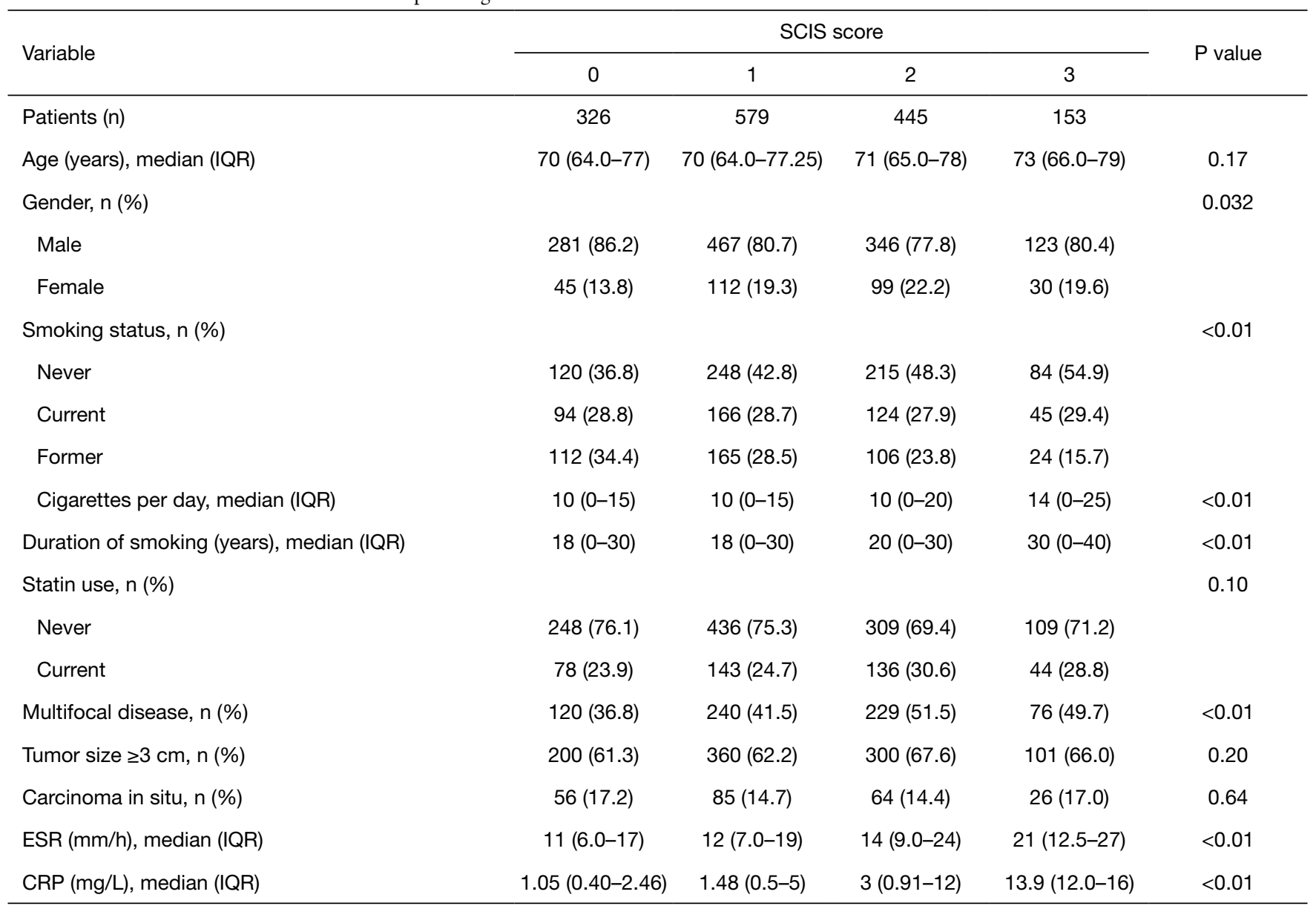

SCIS, systemic cumulative inflammation score; ESR, erythrocyte sedimentation rate; CRP, C-reactive protein.

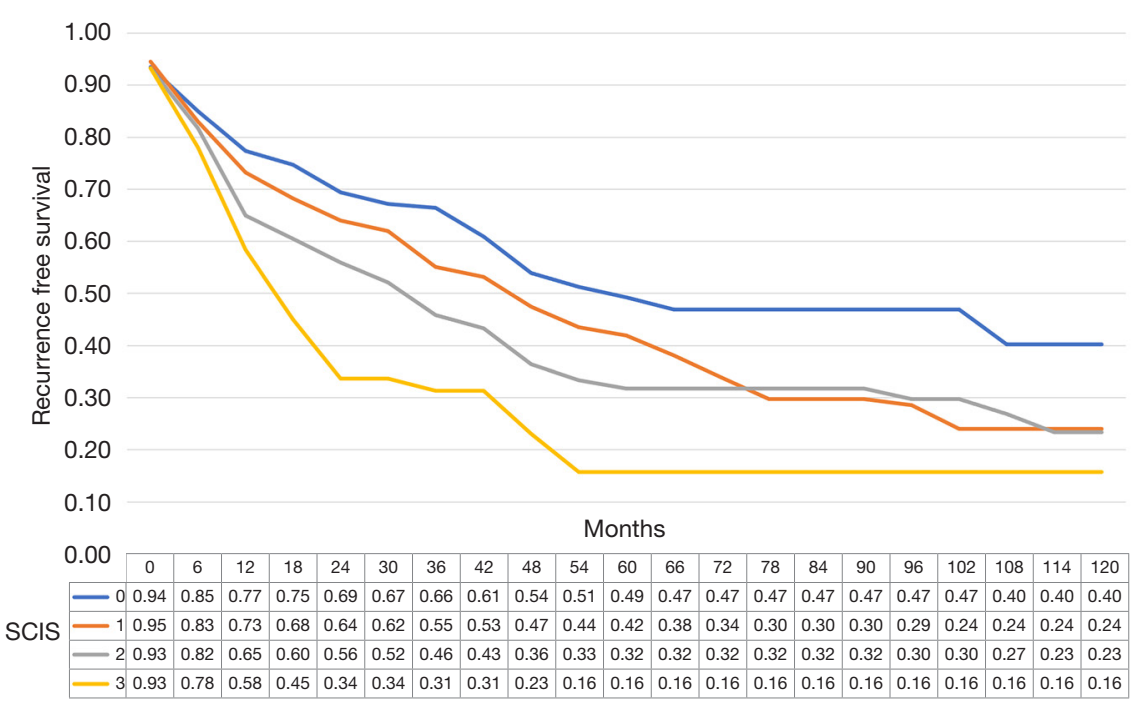

Figure 1 Recurrence survival estimates according to SCIS. SCIS, systemic combined inflammatory score. 


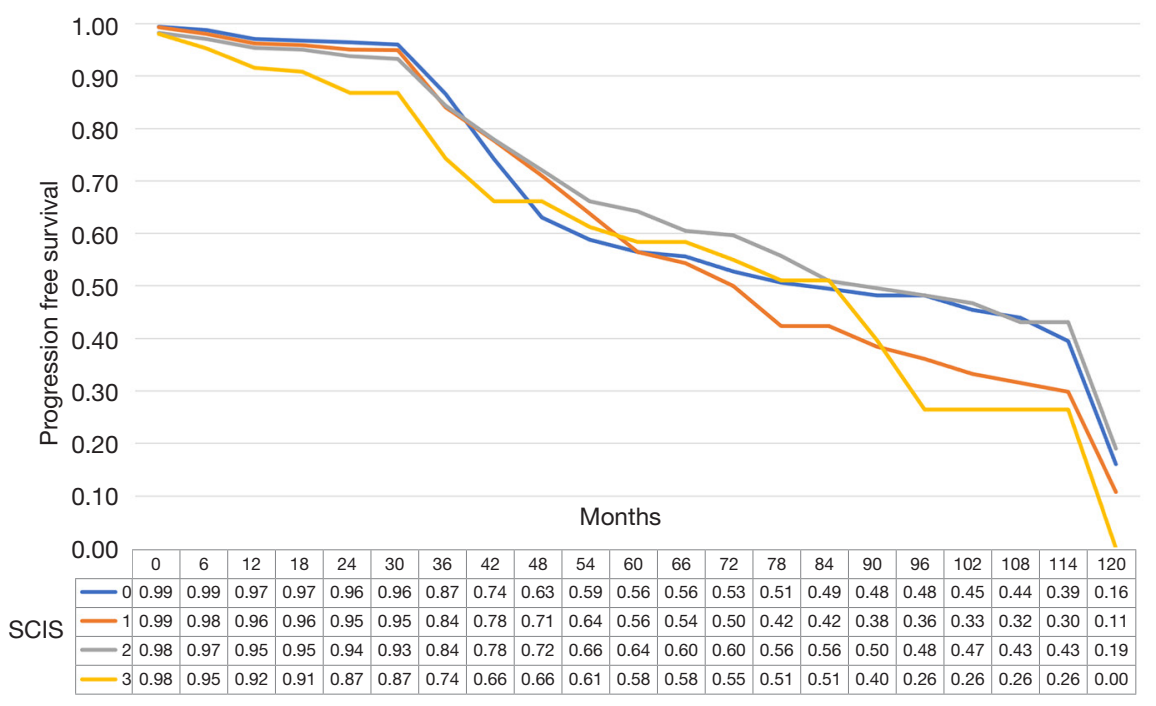

Figure 2 Progression free estimates according to SCIS. SCIS, systemic combined inflammatory score.

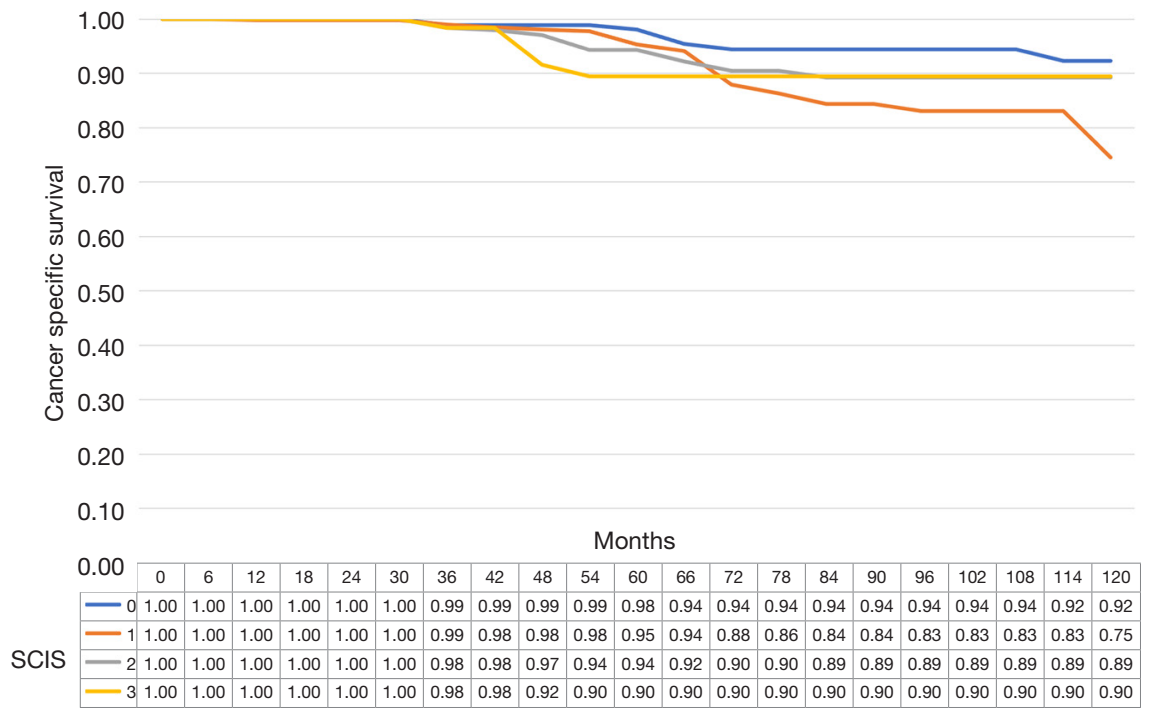

Figure 3 Cancer specific survival estimates according to SCIS. SCIS, systemic combined inflammatory score.

recurrence or progression, in order to establish the optimal follow-up and treatment strategies.

The EORTC risk tables are the most used tool for risk stratification and prognosis prediction in NMIBC patients. The EAU risk categories are a possible alternative to the EORTC risk tables and can be used for comparable risk stratification (12). The CUETO scoring model is more accurate than the EORTC risk tables, in a subgroup of NMIBC patients treated with BCG (4). Unfortunately, the biggest problem we find in the stratification of patients is the fact that they are treated differently from the current standard. Furthermore, evaluation of tumor size and other variables are subjective. For these reasons, risk stratification and prognosis estimation should be performed when NMIBC is diagnosed. The lack of standardization makes stratification difficult and imprecise and this has a shortterm impact on the patient's treatment and follow-up, and in the long term on the patient's quality of life and 


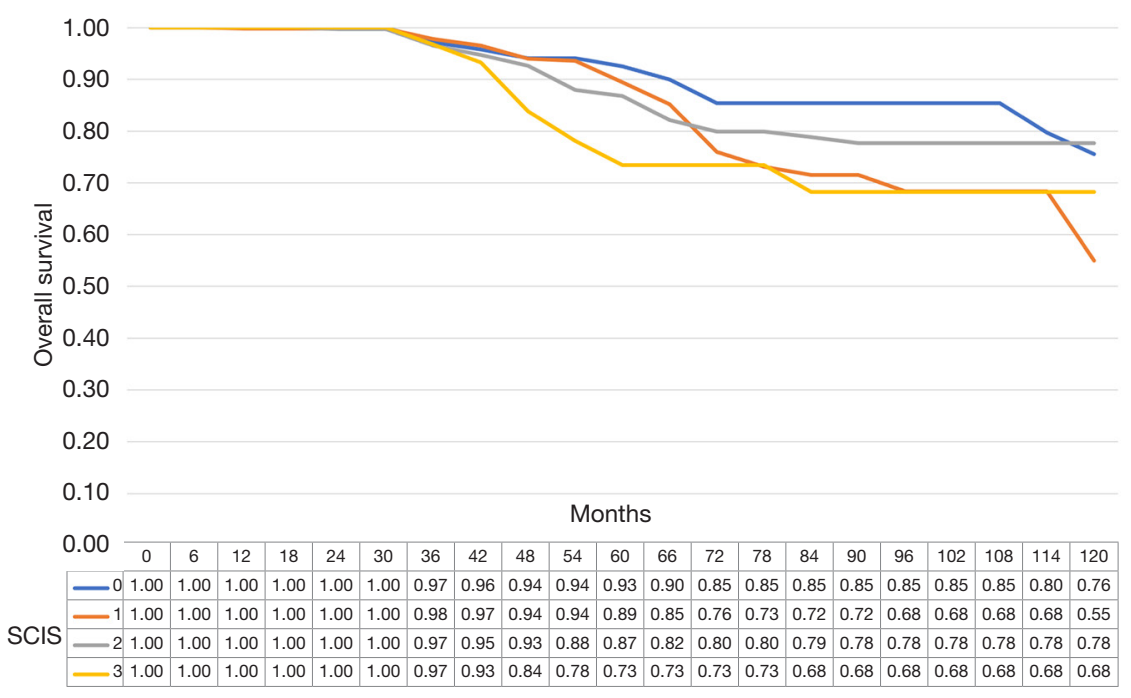

Figure 4 Overall survival estimates according to SCIS. SCIS, systemic combined inflammatory score.

Table 3 Multivariable Cox-regression analysis for recurrence-free survival, progression-free survival, cancer-specific mortality, and overall mortality

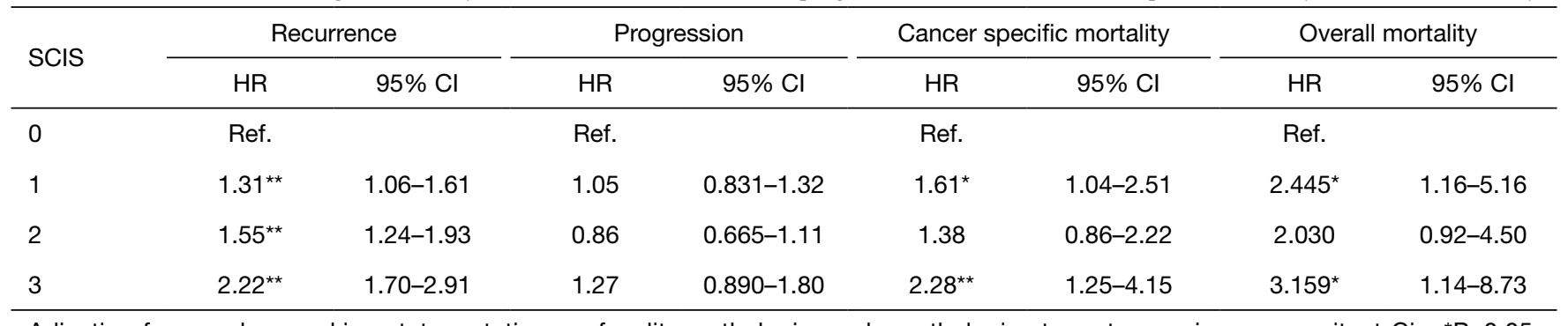

Adjusting for: gender, smoking status, statin use, focality, pathologic grade, pathologic stage, tumor size, concomitant Cis. ${ }^{*} \mathrm{P}<0.05$; ${ }^{\star *} \mathrm{P}<0.01$. SCIS, systemic combined inflammatory score; HR, hazard ratio; $\mathrm{Cl}$, confidence interval.

oncological outcomes. All this can be overcome only if we are able to find clinical or even better molecular and genetic markers to predict oncological outcomes, before starting the treatment and the reorganization of the disease, which in any case alters its natural history $(13,14)$. Different biomarkers have been used in the past with the aim of calculate response after therapy for BC $(8,15,16)$ and several studies have also confirmed the potential role of flogosis markers in urothelial cancers $(17,18)$.

Based on our results, cigarette smoking may affect systemic immune and inflammation marker levels. Previous data in fact have reported that systemic inflammatory response triggered by exposure to smoking is characterized by the stimulation of the hematopoietic system, specifically bone marrow, that determines the increase of leukocytes and platelets into the circulation, due to the relative increase in polymorphonuclear neutrophil counts in the circulation of smokers, and smoking also influences the proliferation and death pathways of lymphocytes $(19,20)$.

For all these reasons, we hypothesize that marker of inflammation could serve as predictors of BC outcomes.

A study from Cantiello et al. has previously demonstrated that subjects with higher systemic inflammatory markers (SIM) were at more risk to recur and progress (8). In the same way the Glasgow prognostic score (GPS) and mGPS, an inflammation-based model, has been shown to be an accurate predictor of prognosis in colorectal cancer patients, non-small-cell lung cancer, pancreatic cancer, esophageal cancer, and hepatocellular carcinoma in several studies (21-24). Furthermore, it has been shown that the preoperative PNI is also associated with clinical outcomes in patients with malignant tumors. In fact, a higher PNI has 
been proven to be associated with a favorable outcome in glioblastomas patients, while a low PNI has been associated with poor survival in lung cancer $(25,26)$. Our data agree with current literature. In fact, according to our study, high PNI levels have a positive effect on the OS, unlike the mGPS and the SIM score which, if with a higher value, correlates negatively with the OS. By combining these 3 biomarkers in the SCIS we can predict patients more accurately than might benefit of a more incisive treatment. In fact, according to our study, high SCIS correlates with a greater risk to recur or die for cancer or any disease.

As regards to NMIBC patients, Kimura et al. showed in a retrospective analysis of above one-thousand cases that patients with elevated mGPS were more likely to experience disease recurrence and progression (27). Furthermore, the addition of mGPS to a standard prognostic model for prediction of disease progression improved its discrimination by $2.2 \%$. This was confirmed also in patients with muscle-invasive disease: in a retrospective study of one-thousand patients treated with radical cystectomy, the mGPS was independently associated with RFS (28). In another little case-report series, PNI was shown to be significantly associated with predicting tumour recurrence in NMIBC (29).

Indeed, to our knowledge, this is the first study that in a single score, namely the SCIS, are merged the mGPS, the SIM score, and the PNI.

However, this study is not devoid of limitations. First, this is a retrospective study, which may have led to a selection bias. Furthermore, we could not determine the experience of each surgeon, the eventual use of novel endoscopic visualization techniques. This study is also limited by the lack of standardization of the surgical procedures, adjuvant intravesical instillation therapy and follow-up scheduling. Finally, the model should need an external validation in a different cohort and outside Europe and corroborate the comparison with SIM and other scores.

Despite these limitations, we elucidated the relationship between the mGPS score, the SIM score and the PNI and the clinicopathological characteristics and between the SCIS and oncological outcomes in above 1,500 patients. This score can be easily calculated and requires routine blood examination before surgery. Indeed, the present study offers several opportunities for future research in this field: indeed, multicenter prospective studies should provide external validation of the SCIS in different clinical scenarios and integrate our model in a more comprehensive decision- making tool to individualize adjuvant treatment after TURB and eventually select those patients who might benefit of an early radical treatment. Thus, predictive markers are essential in BC also in order to identify those patients in which an early cystectomy could be considered beneficial.

\section{Conclusions}

Risk stratification and prognostic models are fundamental because they allow for adaptation of treatment, followup and future development. The turning point lies in the identification of precise biomarkers that measure the intrinsic biological potential of the tumor and the patient. In this regard, the inclusion of the predictive models of inflammation in clinical practice may help improve the prediction of cancer outcomes or experience disease mortality.

\section{Acknowledgments}

The authors would like to express their deepest gratitude to Fondazione Muto Onlus in Naples for the support of the publication of this manuscript.

Funding: None.

\section{Footnote}

Reporting Checklist: The authors have completed the REMARK reporting checklist. Available at http://dx.doi. org/10.21037/tau-20-1272

Data Sharing Statement: Available at http://dx.doi. org/10.21037/tau-20-1272

Conflicts of Interest: All authors have completed the ICMJE uniform disclosure form (available at http://dx.doi. org/10.21037/tau-20-1272). The authors have no conflicts of interest to declare.

Ethical Statement: The authors are accountable for all aspects of the work in ensuring that questions related to the accuracy or integrity of any part of the work are appropriately investigated and resolved. The study was conducted in accordance with the Declaration of Helsinki (as revised in 2013). The study was approved by institutional ethics board of Policlinico Hospital Federico II of Naples (No.: 310/2018) and informed consent was taken from all the patients. 
Open Access Statement: This is an Open Access article distributed in accordance with the Creative Commons Attribution-NonCommercial-NoDerivs 4.0 International License (CC BY-NC-ND 4.0), which permits the noncommercial replication and distribution of the article with the strict proviso that no changes or edits are made and the original work is properly cited (including links to both the formal publication through the relevant DOI and the license). See: https://creativecommons.org/licenses/by-nc-nd/4.0/.

\section{References}

1. Antoni S, Ferlay J, Soerjomataram I, et al. Bladder Cancer Incidence and Mortality: A Global Overview and Recent Trends. Eur Urol 2017;71:96-108.

2. Mariappan P, Smith G. A surveillance schedule for G1Ta bladder cancer allowing efficient use of check cystoscopy and safe discharge at 5 years based on a 25 -year prospective database. J Urol 2005;173:1108-11.

3. Sylvester RJ, van der Meijden APM, Oosterlinck W, et al. Predicting recurrence and progression in individual patients with stage Ta T1 bladder cancer using EORTC risk tables: a combined analysis of 2596 patients from seven EORTC trials. Eur Urol 2006;49:466-5; discussion 475-7.

4. Fernandez-Gomez J, Madero R, Solsona E, et al. Predicting nonmuscle invasive bladder cancer recurrence and progression in patients treated with bacillus Calmette-Guerin: the CUETO scoring model. J Urol 2009;182:2195-203.

5. Busato Júnior WFS, Almeida GL, Ribas CAPM, et al. EORTC Risk Model to Predict Progression in Patients With Non-Muscle-Invasive Bladder Cancer: Is It Safe to Use in Clinical Practice? Clin Genitourin Cancer 2016;14:176-82.

6. Jobczyk M, Stawiski K, Fendler W, et al. Validation of EORTC, CUETO, and EAU risk stratification in prediction of recurrence, progression, and death of patients with initially non-muscle-invasive bladder cancer (NMIBC): A cohort analysis. Cancer Med 2020;9:4014-25.

7. Karl A, Adejoro O, Saigal C, et al. General adherence to guideline recommendations on initial diagnosis of bladder cancer in the United States and influencing factors. Clin Genitourin Cancer 2014;12:270-7.

8. Cantiello F, Russo GI, Vartolomei MD, et al. Systemic Inflammatory Markers and Oncologic Outcomes in Patients with High-risk Non-muscle-invasive Urothelial Bladder Cancer. Eur Urol Oncol 2018;1:403-10.
9. Leitch EF, Chakrabarti M, Crozier JEM, et al. Comparison of the prognostic value of selected markers of the systemic inflammatory response in patients with colorectal cancer. Br J Cancer 2007;97:1266-70.

10. Lee JY, Kim HI, Kim YN, et al. Clinical Significance of the Prognostic Nutritional Index for Predicting Shortand Long-Term Surgical Outcomes After Gastrectomy: A Retrospective Analysis of 7781 Gastric Cancer Patients. Medicine (Baltimore) 2016;95:e3539.

11. Luque-Fernandez MA, Redondo-Sánchez D, Maringe C. cvauroc: Command to compute cross-validated area under the curve for ROC analysis after predictive modeling for binary outcomes. Stata J 2019;19:615-25.

12. Rieken M, Shariat SF, Kluth L, et al. Comparison of the EORTC tables and the EAU categories for risk stratification of patients with nonmuscle-invasive bladder cancer. Urol Oncol 2018;36:8.e17-8.e24.

13. Creighton CJ. The clinical applications of The Cancer Genome Atlas project for bladder cancer. Expert Rev Anticancer Ther 2018;18:973-80.

14. Campi R, Seisen T, Roupret M. Unmet Clinical Needs and Future Perspectives in Non-muscle-invasive Bladder Cancer. Eur Urol Focus 2018;4:472-80.

15. Ferro M, Vartolomei MD, Russo GI, et al. An increased body mass index is associated with a worse prognosis in patients administered BCG immunotherapy for T1 bladder cancer. World J Urol 2019;37:507-14.

16. Vartolomei MD, Ferro M, Cantiello F, et al. Validation of Neutrophil-to-lymphocyte Ratio in a Multi-institutional Cohort of Patients With T1G3 Non-muscle-invasive Bladder Cancer. Clin Genitourin Cancer 2018;16:445-52.

17. Ferro M, Di Lorenzo G, Vartolomei MD, et al. Absolute basophil count is associated with time to recurrence in patients with high-grade T1 bladder cancer receiving bacillus Calmette-Guérin after transurethral resection of the bladder tumor. World J Urol 2020;38:143-50.

18. Ferro M, Di Lorenzo G, Buonerba C, et al. Predictors of Residual T1 High Grade on Re-Transurethral Resection in a Large Multi-Institutional Cohort of Patients with Primary T1 High-Grade/Grade 3 Bladder Cancer. J Cancer 2018;9:4250-4.

19. Ogihara K, Kikuchi E, Yuge K, et al. The Preoperative Neutrophil-to-lymphocyte Ratio is a Novel Biomarker for Predicting Worse Clinical Outcomes in Non-muscle Invasive Bladder Cancer Patients with a Previous History of Smoking. Ann Surg Oncol 2016;23:1039-47.

20. Arnson Y, Shoenfeld Y, Amital H. Effects of tobacco smoke on immunity, inflammation and autoimmunity. J 
Autoimmun 2010;34:J258-65.

21. Choi KW, Hong SW, Chang YG, et al. Inflammationbased score (Glasgow prognostic score) as an independent prognostic factor in colorectal cancer patients. Ann Surg Treat Res 2014;86:309-13.

22. Forrest LM, McMillan DC, McArdle CS, et al. Evaluation of cumulative prognostic scores based on the systemic inflammatory response in patients with inoperable nonsmall-cell lung cancer. Br J Cancer 2003;89:1028-30.

23. Kurahara H, Maemura K, Mataki Y, et al. Prognostication by inflammation-based score in patients with locally advanced pancreatic cancer treated with chemoradiotherapy. Pancreatology 2015;15:688-93.

24. Crumley ABC, McMillan DC, McKernan M, et al. Evaluation of an inflammation-based prognostic score in patients with inoperable gastro-oesophageal cancer. Br J Cancer 2006;94:637-41.

25. Ding JD, Yao K, Wang PF, et al. Clinical significance of prognostic nutritional index in patients with glioblastomas.

Cite this article as: Ferro M, Di Mauro M, Cimino S, Morgia G, Lucarelli G, Abu Farhan AR, Vartolomei MD, Porreca A, Cantiello F, Damiano R, Busetto GM, Del Giudice F, Hurle $\mathrm{R}$, Perdonà $\mathrm{S}$, Borghesi $\mathrm{M}$, Bove $\mathrm{P}$, Autorino $\mathrm{R}$, Crisan $\mathrm{N}$, Marchioni M, Schips L, Soria F, Mari A, Minervini A, Veccia A, Battaglia M, Terracciano D, Musi G, Cordima G, Muto M, Mirone V, de Cobelli O, Russo GI. Systemic combining inflammatory score (SCIS): a new score for prediction of oncologic outcomes in patients with high-risk non-muscleinvasive urothelial bladder cancer. Transl Androl Urol 2021;10(2):626-635. doi: 10.21037/tau-20-1272
Medicine (Baltimore) 2018;97:e13218.

26. Wang Z, Wang Y, Zhang X, et al. Pretreatment prognostic nutritional index as a prognostic factor in lung cancer: Review and meta-analysis. Clin Chim Acta 2018;486:303-10.

27. Kimura S, D'Andrea D, Soria F, et al. Prognostic value of modified Glasgow Prognostic Score in non-muscleinvasive bladder cancer. Urol Oncol 2019;37:179.e19179.e28.

28. Ferro M, De Cobelli O, Buonerba C, et al. Modified Glasgow Prognostic Score is Associated With Risk of Recurrence in Bladder Cancer Patients After Radical Cystectomy: A Multicenter Experience. Medicine (Baltimore) 2015;94:e1861.

29. Cui J, Chen S, Bo Q, et al. Preoperative prognostic nutritional index and nomogram predicting recurrencefree survival in patients with primary non-muscle-invasive bladder cancer without carcinoma in situ. Onco Targets Ther 2017;10:5541-50. 


\section{Supplementary}

Table S1 Association between baseline clinicopathologic characteristics and cumulative mGPS score in the total cohort

\begin{tabular}{|c|c|c|c|c|}
\hline & \multicolumn{3}{|c|}{ Modified Glasgow Prognostic Score } & $P$ value \\
\hline Patients (n) 1510 & 1081 & 387 & 42 & \\
\hline Median age, yr (IQR) & $71(65-78)$ & $70(65-78)$ & $70(63.75-79.25)$ & 0.82 \\
\hline Gender, n (\%) & & & & 0.27 \\
\hline Female & $205(19.0)$ & $71(18.3)$ & $12(28.6)$ & \\
\hline Smoking status, n (\%) & & & & $<0.01$ \\
\hline Never & $455(42.1)$ & $202(52.2)$ & $16(38.1)$ & \\
\hline Current & $306(28.3)$ & $107(27.6)$ & $16(38.1)$ & \\
\hline Median smoking years, n (IQR) & $18(0-30)$ & $25(0-35)$ & $25(0-40)$ & $<0.01$ \\
\hline Statin use, n (\%) & & & & 0.02 \\
\hline Never & $773(71.5)$ & $305(78.8)$ & $30(71.4)$ & \\
\hline Current & $308(28.5)$ & $82(21.2)$ & $12(28.6)$ & \\
\hline Multifocal disease, $\mathrm{n}(\%)$ & $449(41.5)$ & $199(51.4)$ & $21(50.0)$ & $<0.01$ \\
\hline Tumor size $\geq 3 \mathrm{~cm}, \mathrm{n}(\%)$ & $670(62.0)$ & $271(70.0)$ & $26(63.4)$ & 0.02 \\
\hline Carcinoma in situ, $\mathrm{n}(\%)$ & $169(15.6)$ & $57(14.7)$ & $5(11.9)$ & 0.75 \\
\hline Median ESR, mm/h (IQR) & $12(7-20)$ & $17(10.15-26)$ & $20.50(12.75-27)$ & $<0.01$ \\
\hline
\end{tabular}


Table S2 Association between baseline clinicopathologic characteristics and cumulative PNI in the total cohort

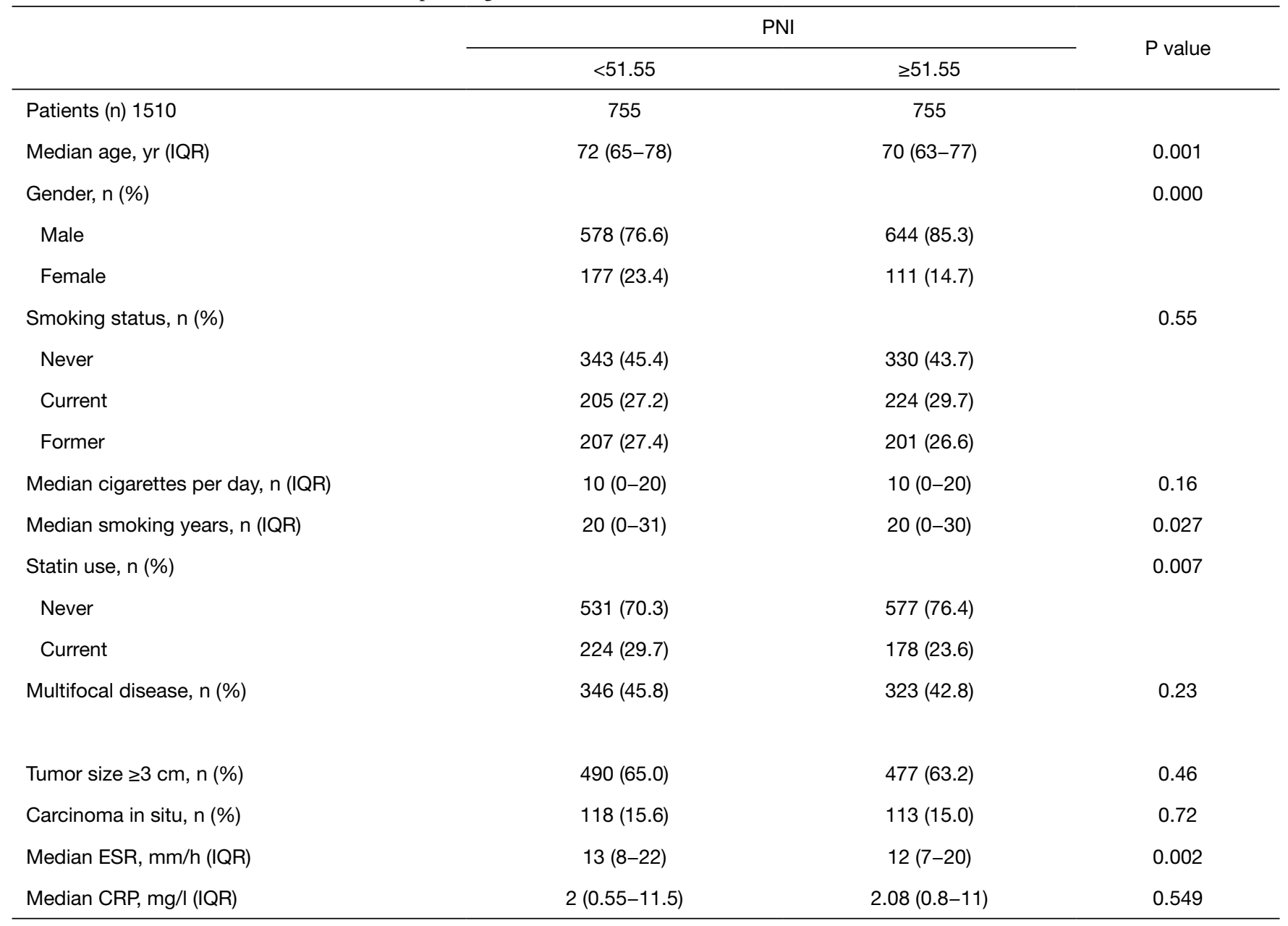


Table S3 Association between baseline clinicopathologic characteristics and cumulative SIM score in the total cohort

\begin{tabular}{|c|c|c|c|c|c|}
\hline & \multicolumn{4}{|c|}{ SIM } & $P$ value \\
\hline Patients (n) 1510 & 150 & 603 & 610 & 140 & \\
\hline Median age, yr (IQR) & $73(65-78)$ & $70(64-77)$ & $71(65-78)$ & $70(67-80)$ & 0.14 \\
\hline Gender, n (\%) & & & & & 0.17 \\
\hline Female & $23(15.3)$ & $120(19.9)$ & $124(20.3)$ & $19(13.6)$ & \\
\hline Smoking status, n (\%) & & & & & 0.000 \\
\hline Never & $51(34.0)$ & $242(40.1)$ & $297(48.7)$ & $77(55.0)$ & \\
\hline Current & $43(28.7)$ & $165(27.4)$ & $181(29.7)$ & $40(28.6)$ & \\
\hline Median smoking years, n (IQR) & $15(0-30)$ & $19.50(0-30)$ & $25(0-35)$ & $20(0-35)$ & 0.035 \\
\hline Statin use, n (\%) & & & & & 0.006 \\
\hline Never & $119(79.3)$ & $462(76.6)$ & $427(70.0)$ & $94(67.1)$ & \\
\hline Current & $31(20.7)$ & $141(23.4)$ & $183(30.0)$ & $46(32.9)$ & \\
\hline Multifocal disease, $\mathrm{n}(\%)$ & $70(46.7)$ & $231(38.3)$ & $299(49.0)$ & $65(46.4)$ & 0.002 \\
\hline Tumor size $\geq 3 \mathrm{~cm}, \mathrm{n}(\%)$ & $113(75.3)$ & $367(61.0)$ & $398(65.2)$ & $83(59.3)$ & 0.006 \\
\hline Carcinoma in situ, n (\%) & $17(11.3)$ & $103(17.1)$ & $83(13.6)$ & $28(20.0)$ & 0.07 \\
\hline Median ESR, mm/h (IQR) & $8.75(6-14.25)$ & $12(7.80-20)$ & $14(8-24.25)$ & $16(8-25)$ & 0.000 \\
\hline
\end{tabular}
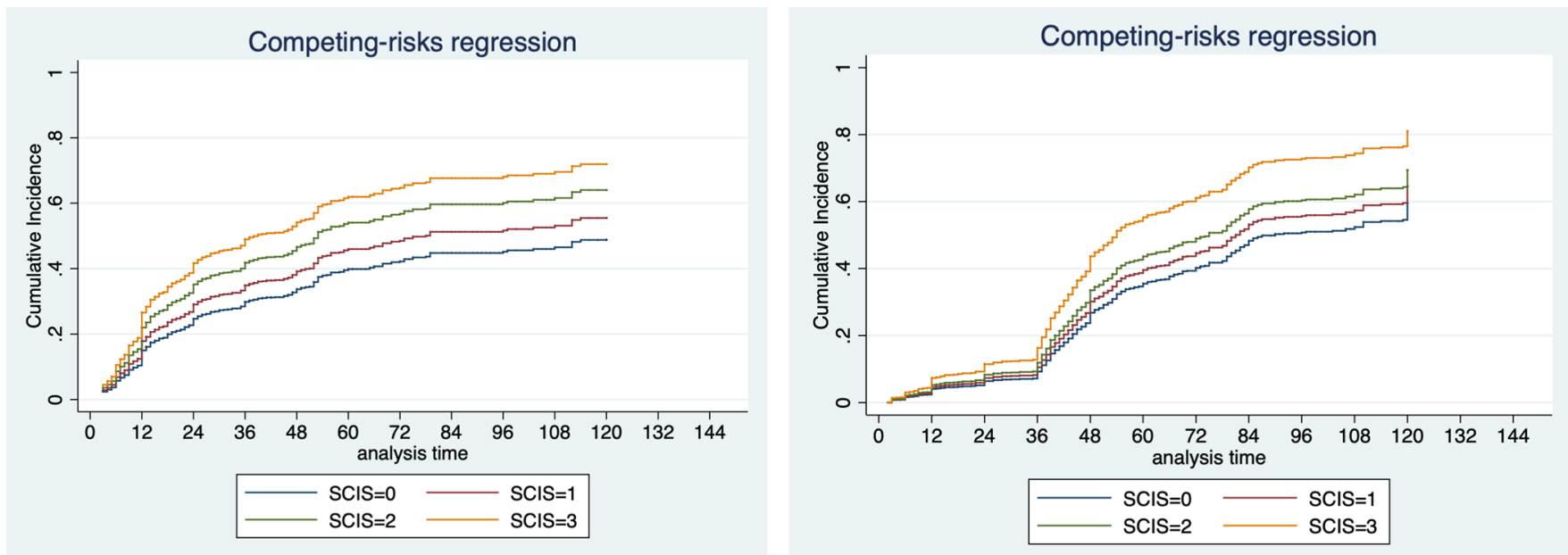

Figure S1 Fine-Gray competing risk analysis of recurrence (a) and progression (b) according to SCIS. 


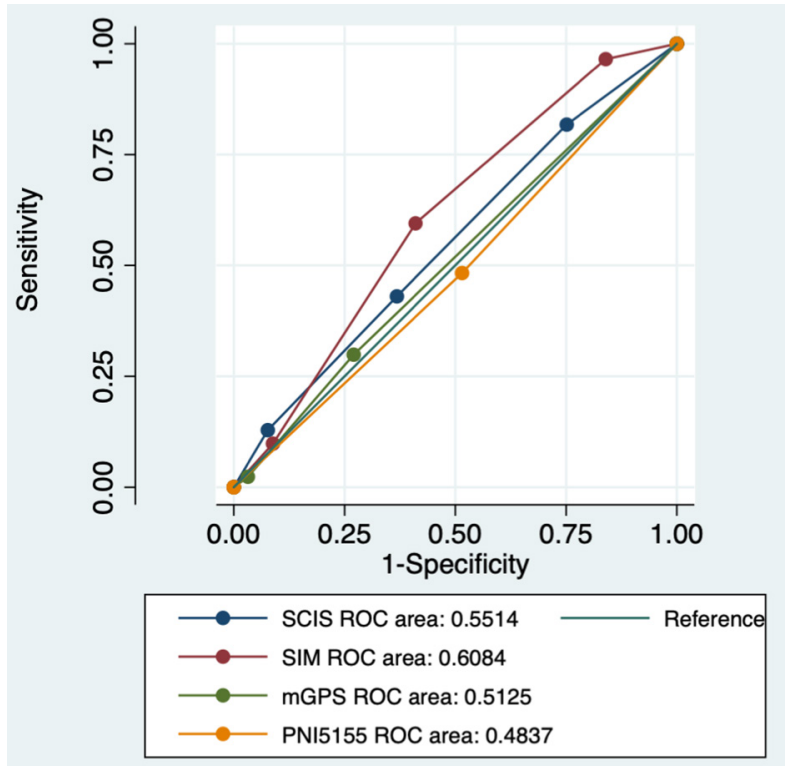

Figure S2 ROC for recurrence prediction for SIM, SCIS, mGPS and PNI. 